\title{
Mathematical modelling of liquid meniscus shape in cylindrical micro-channel for normal and micro gravity conditions
}

\author{
Igor Marchuk and Yuriy Lyulin* \\ Institute of Thermophysics, 1, Lavrentiev Ave., 630090, Novosibirsk, Russia \\ Novosibirsk State University, 2, Pirogov Ave., 630090, Novosibirsk, Russia
}

\begin{abstract}
Mathematical model of liquid meniscus shape in cylindrical micro-channel of the separator unit of condensing/separating system is presented. Moving liquid meniscus in the $10 \mu \mathrm{m}$ cylindrical microchannel is used as a liquid lock to recover the liquid obtained by condensation from the separators. The main goal of the liquid locks to prevent penetration of a gas phase in the liquid line at the small flow rate of the condensate and because of pressure fluctuations in the vapor-gas-liquid loop. Calculation of the meniscus shape has been performed for liquid FC-72 at different values of pressure difference gas - liquid and under normal and micro gravity conditions.
\end{abstract}

\section{Introduction}

In recent decades, the use of two-phase flows for cooling of electronic components, such as computer chips, power electronics, converter chips and inverters in hybrid cars, powerful lasers, light-emitting diodes, etc., has developed significantly. One of the promising solutions allowing to remove high heat fluxes is technology using processes with phase change, for example evaporation of a thin liquid film moving in a flat micro-channel under the action of a gas flow [1,2]. Often it is of fundamental importance for the film to use an inert (non-condensable) gas. The vapour produced during evaporation is mixed with noncondensable gas. To operate in a closed loop, the vapour should be condensed and the resulting liquid separated from the gas. Further liquid and gas has to be separately sent again to the evaporator for cooling. Thus, the creation of a condensing/separating system is one and the most important task for improving the efficiency of the two-phase cooling system. Specifically it plays a central role in thermal control systems for space application $[3,4]$.

Concept of condenser with liquid separators is shown in Fig. 1. To separate a condensate from a vapor-gas flow two separators are used. Liquid flows from condenser to the slot of the first separator by the tangential stress on the interface and is continuously pumped out by the pump. The gas phase is not completely free of vapour and liquid at this stage. The inert gas flow that contains some residuals of vapour and liquid droplets passes

* Corresponding author: 1yulin@itp.nsc.ru 
through a second separator. This device is used to extract from the gas phase a very small liquid droplets created in the condenser but transported by the inert gas flow beyond the first separator. The so-called "liquid lock" is used to recover the liquid obtained by condensation from the separators.

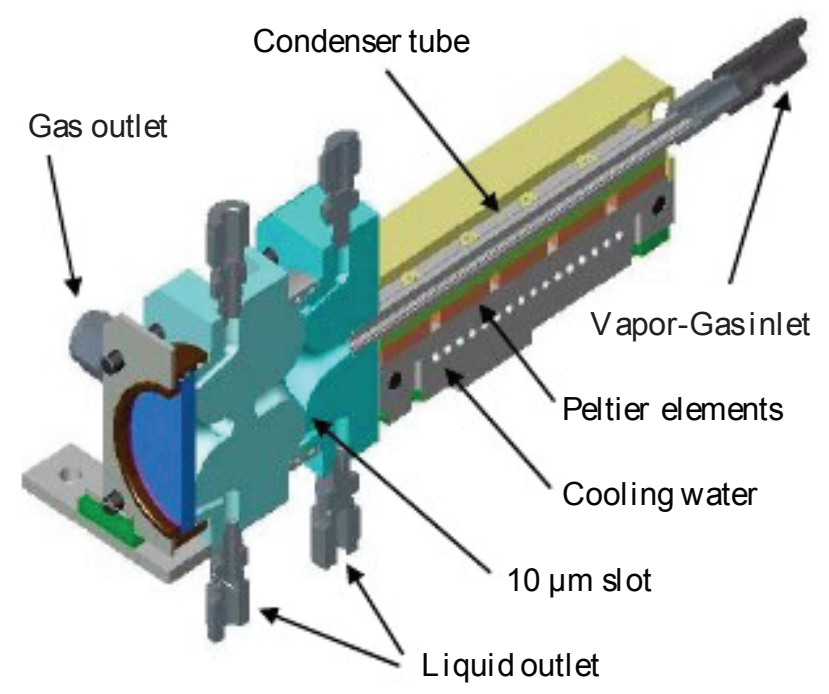

Fig. 1. Cross section of the condensing/separating system.

The main goal of this work is development of a mathematical model of liquid meniscus shape in cylindrical microchannel of the separator unit of condensing/separating system. Moving liquid meniscus is used as the liquid locks to prevent penetration of a gas phase in the liquid line at the small flow rate of the condensate and because of pressure fluctuations in the vapour-gas-liquid loop.

\section{Mathematical model}

The motion of a two-phase flow in a separator is described by the Navier-Stokes equations. The liquid-gas interface is assumed to be stable, since the process is stationary. Assuming that the flow rate of liquid pumped from the separator is relatively small, it can be considered that the velocities of liquid and gas are zero.

Under gravity meniscus shape is not round and it is deformed by hydrostatic pressure. Let find meniscus shape in this case. Wetting contact angle is equal $\gamma$. It means that angle between tangent plane to liquid - gas interface and tangent plane to solid surface in contact point is equal $\gamma$.

Pressure difference between liquid and gas is given by Laplas equation:

$$
\Delta p_{s}=\sigma\left(\frac{1}{r_{m}}+\frac{1}{R_{2}}\right)
$$

In this case $r_{m}<<R_{2}$. It allows to neglect the radial curvature of the interface and pass on two dimensional problem.

Let $x_{m}$ is distance from the beginning the channel widening to meniscus, $r_{m}$ is the meniscus radius, $R_{S}$ is the radius of separator wall, $h$ is a half of separator gap thickness, $R_{i n}$ - inner radius of separator chamber. Real sizes in separator are following: $R_{s}=10 \mathrm{~mm}, R_{\text {in }}$ $=13.35 \mathrm{~mm}$ and $h=0.005 \mathrm{~mm}$, Fig. 2 . 


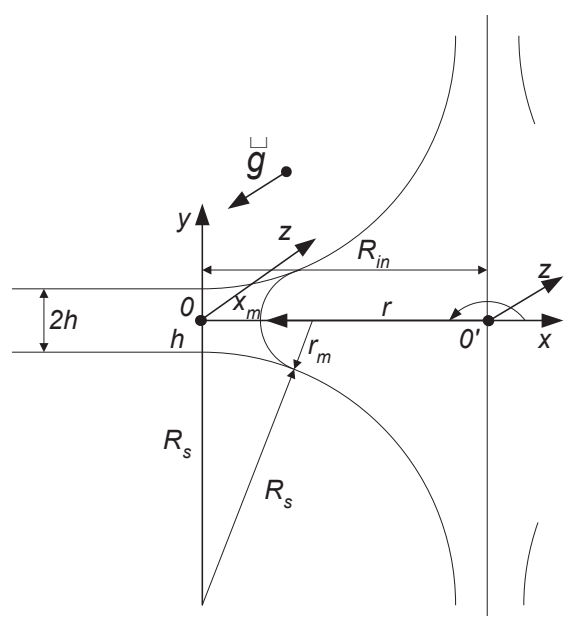

Fig. 2. Geometrical scheme of the meniscus position in separator, axis $z$ directed opposite to the vector of gravitation.

The meniscus radius $r_{m}$ obey the following system of equations:

$$
\begin{cases}x^{2}+\left(y+R_{s}+h\right)^{2}=R_{s}^{2} & \text { solid surface of separator } \\ \left(x-\left(r_{m}+x_{m}\right)\right)^{2}+y=r_{m}^{2} & \text { liquid gas interface (meniscus) } \\ x\left(x-\left(r_{m}+x_{m}\right)\right)+y\left(y+R_{s}+h\right)=-r_{m} R_{s} \cos \gamma & \text { wetting contact angle condition }\end{cases}
$$

Excluding from this system coordinates of the contact point $x, y$ one obtain an expression for radius $r_{m}$ of the meniscus:

$$
r_{m}=\frac{x_{m}^{2}+2 R_{s} h+h^{2}}{2\left(R_{s} \cos \gamma-x_{m}\right)}
$$

Let pressure difference $\Delta p$ is known. At gravity condition it satisfy to the expression

$$
\Delta p=-\frac{\sigma}{r_{m}}+\rho g z-\rho g Z_{d p}
$$

here $\Delta p=p_{l}-p_{g}$ is measured value with detector pressure difference between liquid and gas, $Z_{d p}$ is coordinate $z$ of the pressure difference detector sensor location. Hydrostatic pressure in the gas phase is neglected.

Value of coordinate $z$ of the interface can be expressed through polar angle $\phi$, counting from the positive direction of the axis $x$ in the plane $z x$,

$$
z=r \sin \phi
$$

where $r$ is a radius vector from separator centre

$$
r=R_{i n}-x_{m}
$$

Substitution (2) and (4) into expression for pressure difference (3) gives following equation:

$$
\sin \phi=\frac{\Delta p+\rho g Z_{d p}}{\rho g\left(R_{i n}-x_{m}\right)}+\frac{\sigma}{\rho g} \frac{2\left(R_{s} \cos \gamma-x_{m}\right)}{\left(x_{m}^{2}+2 R_{s} h+h^{2}\right)\left(R_{i n}-x_{m}\right)}
$$


Finally, with taking into account (5)

$$
\sin \phi=\frac{\Delta p+\rho g Z_{d p}}{\rho g r}+\frac{\sigma}{\rho g} \frac{2\left(r-\left(R_{i n}-R_{s} \cos \gamma\right)\right)}{\left(\left(R_{i n}-r\right)^{2}+2 R_{s} h+h^{2}\right) r}
$$

This is the equation of the meniscus shape at gravity condition.

At microgravity ( $\mathrm{g}=0$ ) meniscus shape equation (6) takes the form

$$
\Delta p+\frac{2 \sigma\left(r-\left(R_{i n}-R_{s} \cos \gamma\right)\right)}{\left(\left(R_{i n}-r\right)^{2}+2 R_{s} h+h^{2}\right)}=0
$$

Hence

$$
r=R_{i n}-\frac{\sigma}{\Delta p}-\sqrt{\left(\frac{\sigma}{\Delta p}\right)^{2}-2 h R_{s}-h^{2}-2 \frac{\sigma}{\Delta p} R_{s} \cos \gamma}
$$

Here $r$ is constant and meniscus has the shape of circumference.

\section{Results}

Meniscus shape at different values of pressure difference liquid - gas, given at the level of separator centre is shown in Fig. 3. Solid circumference is interior boundary of separator gap. Calculation of equation (8) is performed for liquid FC72 and $Z_{d p}=0$. One can use dimensionless criterion $K_{d p}=\rho g R_{i n} / \Delta p$ for evaluation of gravitation influence on meniscus shape. At $K_{d p}<1$ the gravitation influence on meniscus shape is weak and meniscus shape is close to circumference. At $K_{d p}$ of order of unity and higher meniscus is flattened from below by the gravity, Fig 3 .
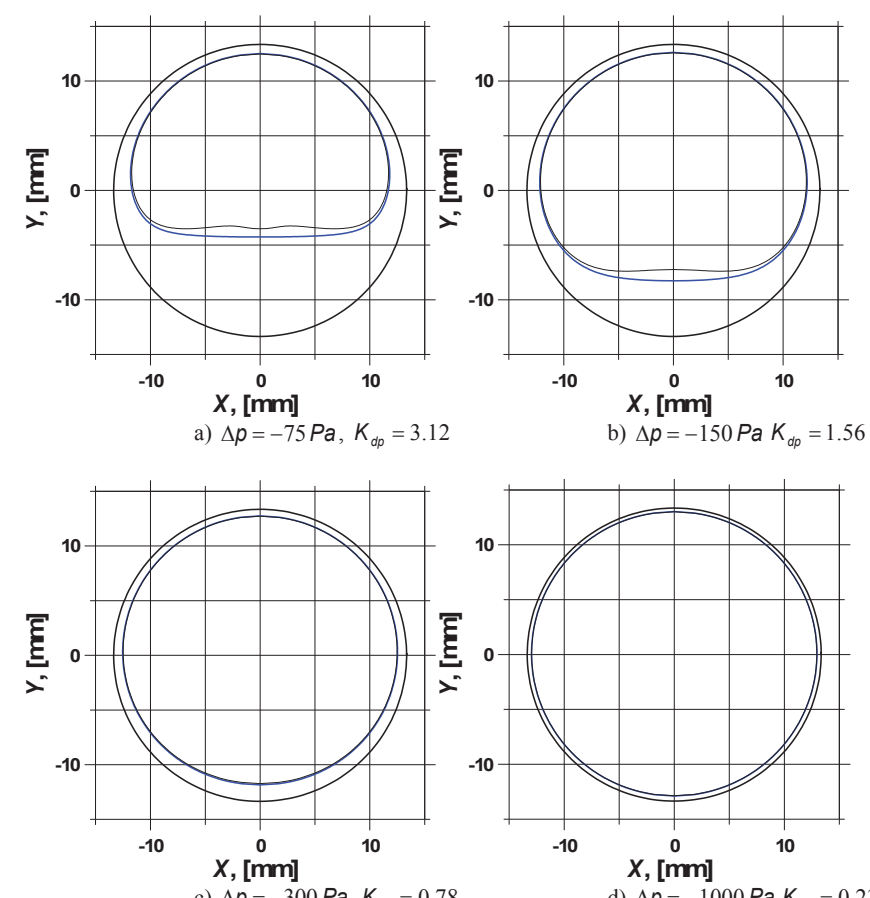

Fig. 3. Meniscus shape at different values of pressure difference liquid - gas. 


\section{Conclusions}

Mathematical model of liquid meniscus shape in cylindrical micro-channel of the separator unit of condensing/separating system has been developed. Moving liquid meniscus in the $10 \mu \mathrm{m}$ cylindrical micro-channel is used as a liquid lock to recover the liquid obtained by condensation from the separators. The main goal of the liquid locks to prevent penetration of a gas phase in the liquid line at the small flow rate of the condensate and because of pressure fluctuations in the vapor-gas-liquid loop. Calculation of the meniscus shape has been performed for liquid FC-72 at different values of pressure difference gas - liquid and under normal and micro gravity conditions.

The authors gratefully acknowledge the support of this work by the Ministry of Education and Science of the Russian Federation (Agreement 14.616.21.0016, project identifier RFMEFI61614X0016).

\section{References}

1. A. Bar-Cohen, P. Wang, ASME. J. Heat Transfer, 134, 051017 (2012)

2. O.A. Kabov, D.V. Zaitsev, V.V. Cheverda, A. Bar-Cohen, Experimental Thermal and Fluid Science, 35, 825 (2011)

3. V. Serin, P. Lavieille, M. Miscevic, Microgravity Science and Technology, 21 (1), 103-109, (2009)

4. I.V. Marchuk and O.A. Kabov, Encyclopedia of Two-Phase Heat Transfer and Flow II: Special Topics and Applications, 3, 5, 133 (2015) 